\title{
BUBBLY CAVITATING FLOW GENERATION AND INVESTIGATION OF ITS EROSIONAL NATURE FOR BIOMEDICAL APPLICATIONS
}

\author{
A. $\mathrm{KOŞAR}^{1},{ }^{*}$, A. AKBAŞ ${ }^{1}$, Ö. ŞAHIN ${ }^{1}$, A. KUBILAY ${ }^{1}$, Ö. ORAL ${ }^{2}$ and D. GÖZÜAÇIK ${ }^{2}$ \\ * Corresponding author: Tel.: ++90 2164839621; Fax: ++90 2164839550; Email: kosara@sabanciuniv.edu \\ 1: Mechatronics Engineering Program, Faculty of Engineering and Natural Sciences, Sabancı University, \\ Istanbul, TURKEY \\ 2: Biomedical Sciences and Bioengineering Program, Faculty of Engineering and Natural Sciences, Sabanc1 \\ University, Istanbul, TURKEY
}

\begin{abstract}
The paper presents a study of the generation of hydrodynamic bubbly cavitation in microchannels to investigate the destructive energy output resulting from this phenomenon and its potential use in biomedical applications. The research performed in this study includes the experimental results from bubbly cavitation experiments and the findings showing the destructive effects of bubbly cavitating flow on selected specimens and cells.

The bubbles caused by hydrodynamic cavitation are highly destructive at the surfaces of the target medium on which they are carefully focused. The resulting destructive energy output could be effectively used for good means such as destroying kidney stones or killing infected cancer cells. Motivated by this potential, the cavitation damage (material removal) to cancerous cells and chalk pieces having similar material properties as calcium phosphate in human bones was investigated. Also the potential of hydrodynamic bubbly cavitation generated at the microscale for biomedical treatments was revealed using the microchannel configuration of a microorifice (with an inner diameter of $0.147 \mathrm{~mm}$ and a length of $1.52 \mathrm{~cm}$ ).
\end{abstract}

Keywords: Hydrodynamic cavitation; Bubbly cavitating flow; Microchannel; Cavitation damage; Biomedical treatment; Cancer cells.

\section{Introduction}

Hydrodynamic cavitation is typically initiated with local static pressure reduction below a critical value. Its effects on many types of turbomachinery have been investigated by numerous researchers summarized in typical articles and standard multiphase flow textbooks [1-4]. It is known that every hydraulic device is susceptible to the damage caused by cavitation once the appropriate cavitating flow conditions occur. In most cases, hydrodynamic cavitation is not desired since it limits the performance of the fluidic system, causes catastrophic damage and flow choking, generates acoustic noise, and lowers efficiency [1]. Due to its consequences and its destructive nature, cavitation and the energy associated with it constitute an important research subject.

There are two main sources for creating cavitating flow: hydrodynamic and ultrasonic sources. Although ultrasonic sources have been the most popular means of generating cavitation in laboratory scale studies, and numerous applications of ultrasonic cavitation (including biomedical applications) do occur [5-6], ultrasonic cavitation applications have suffered from several shortcomings on industrial scale applications due to high frequency ultrasound usage. Moreover, ultrasonic cavitation is not as energy efficient as hydrodynamic cavitation [7-11].

With the emergence of microfluidics hydrodynamic cavitation has been considered as an important alternative to ultrasonic cavitation over the last decade. Pioneering studies on hydrodynamic cavitation in microchannels have been successful in showing the unique properties of cavitating flow at the microscale [12-16].

The use of ultrasonic cavitation in treatment of cancerous tissues has been investigated by various researchers $[5-6,17-$ $18]$ and has been widely employed in. It is a non-invasive treatment, where some difficulties are faced in targeting the precise location (sick tissue, kidney stone) of the treatment. Hydrodynamic cavitation is another candidate with a cost effective and energy efficient solution. Moreover, with an 
appropriate delivery system, bubbles produced by cavitating flow could be targeted right to the desired spot, so that the destructive nature of bubbly cavitating flows could provide the treatment of the sick tissue. The current study is designed to explore the feasibility of this treatment method. In it the destructive effect of hydrodynamic cavitation is investigated and checked for controllability and success in the above-mentioned targeting.

This would confirm its suitability for biomedical treatments (e.g. of cancerous tissue and/or kidney stones).

\section{Motivation and Experimental Apparatus and Procedure}

\subsection{Motivation and Theory}

Osborne Reynolds was one of the first researchers who focused on the subject of cavitation, by trying to explain the unusual behavior of ship propellers. These were vulnerable to damage because of their high rotational speeds. What Reynolds achieved was to explain the phenomenon in terms of the possible creation of air bubbles near the propeller blades. In general we understand hydrodynamic cavitation as the phenomenon of formation and growth of vapour or air pockets in fluid flows as a result of local static pressure reduction below a critical value. Cavitation bubble collapse leads to a high energy outcome, thereby generating highly localized, large amplitude shock waves $[1,4$, 19]. Such cavitating flows could be initiated using a successful microchannel and microorifice design. In contrast to macroscale applications such as to propellers, this study. does not involve any moving parts. However, using the same concept of reducing the static pressure, it is proposed to generate cavitation inside a microchannel exit and microorifice. As the fluid passes through the orifice throat, the velocity of the fluid increases in consistency with the Bernoulli equation (with the assumption of no frictional losses through the orifice and neglecting frictional losses through the orifice):

$\frac{P_{1}}{\rho}+\frac{1}{2} V_{1}^{2}=\frac{P_{2}}{\rho}+\frac{1}{2} V_{2}^{2}$
As a result, local static pressure of the fluid decreases. If the pressure decreases to a certain critical value under suitable conditions, vapor starts to form inside the fluid. As the fluid passes through the orifice throat, the pressure starts to increase again, which results in consequent growth of cavitation nuclei in the form of vapour and gas-filled bubble/bubble clouds [14]. Just after the throat is the exit area where the bubbly cavitation could be observed. In Fig. 1, the microchannel configuration with the orifice throat and exit area is shown to provide an idea about the cavitating system.

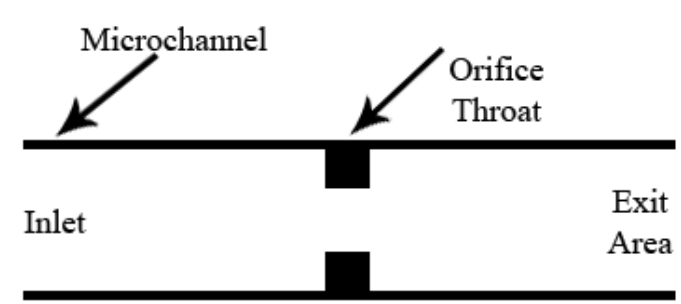

Fig. 1. Microchannel configuration with the orifice throat and exit area.

The bubbles caused by hydrodynamic bubbly cavitation are highly destructive on surfaces once they are localized. Thus, if they could be fine-controlled, they could be utilized for a variety of treatments such as destroying kidney stones or killing infected cancer cells.

The cavitation number, $\sigma$, is a dimensionless number used for quantifying similar cavitating conditions and for representing the intensity of cavitation. It is defined as:

$$
\sigma=\frac{P_{\infty}-P_{v}}{\frac{1}{2} \rho V_{t h}^{2}}
$$

A reduction in cavitation number will increase the intensity and the extent of the cavitation. The channel geometry also affects the formation of cavitation. It is critical not to reduce the cavitation number too much since a transition from bubbly cavitation to supercavitation could occur. This would be unsuitable for the purpose of the current study which is to generate continuous bubbly cavitating flow by designing a microfluidic 
device (bubble generator), and then to expose the emerging bubbles to a small target area (a piece of chalk as a model of a kidney stone or a small area of sick tissue), making observations of the changes in this area.

\subsection{Experimental Setup and Procedure}

A schematic of experimental apparatus is shown in Figs. 2a and 2b. Sequential images of bubble growing and collapse were captured by a microscope and CCD camera unit. The volume flow rates through the microorifice were measured with a flowmeter. The inlet channel, which was also used as tubing connection between the fluidic components (filter, valves, etc.), is made of PVC allowing flexibility and visualization.

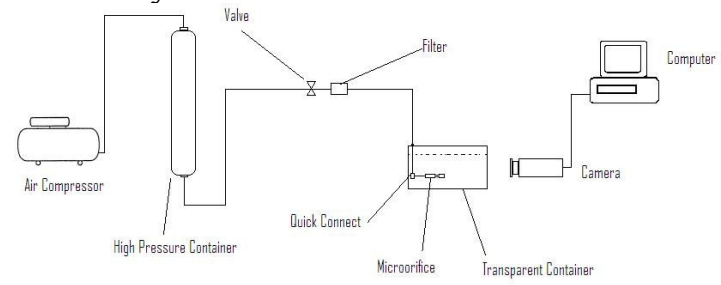

(a)

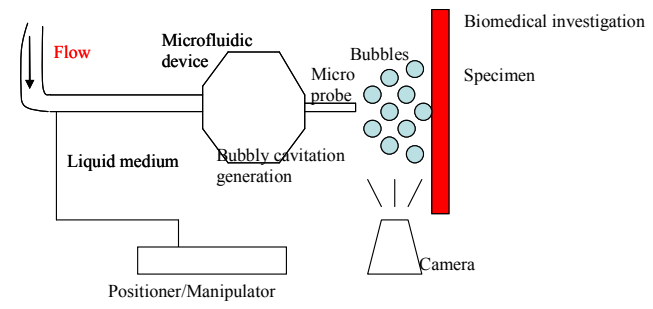

(b)

Fig. 2. (a) Experimental Apparatus, (b) Schematic of Exposure of Bubbly Cavitation

The size of the transparent container (aquarium) used in the setup is $40 \times 40 \times 30 \mathrm{~cm}$. The cavitation is created by a microorifice, which is a plain microchannel of inner diameter of $147 \mu \mathrm{m}$; it is connected to the external tubing from both sides. The system is depicted in Fig. 3. The test setup consists of an air compressor, a high pressure tank, a filter, a pressure gauge, tubings, flow meter, and a fine control valve. The tank was used as a container for deionized water and the compressor/Nitrogen tank was connected to the tank in order to maintain input pressure. The filter was employed to prevent particles larger than $15 \mu \mathrm{m}$.

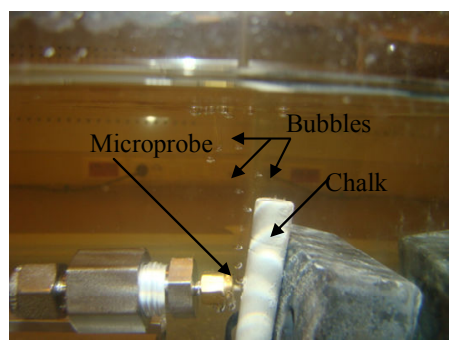

Fig. 3. Picture of the Experimental Apparatus

The tests were conducted by applying different inlet pressure values at the inlet. The maximum pressure applied at the inlet was 7-10 atm, while the outlet pressure was set constant to $1 \mathrm{~atm}$ during tests to better simulate the case for biomedical applications inside a body. The flow rate was controlled with a fine-metering valve. Various inlet pressure values were applied during the tests in order to observe the result at increasing pressure differences until bubbly cavitating flow pattern is obtained.

Examination of bubble sizes in DI water resulted in occurrences of bubble diameters of $60 \mu \mathrm{m}$ to $340 \mu \mathrm{m}$ using a CCD camera. Fig. 4 displays samples of the formed bubbles which grew to different sizes. The uncertainties in pressure and flowrate measurements are $\pm 0.5 \%$ (of entire range) and $\pm 1 \%$ (of measured value), while the uncertainty in the inner diameter measurement was $\pm 2 \mu \mathrm{m}$.

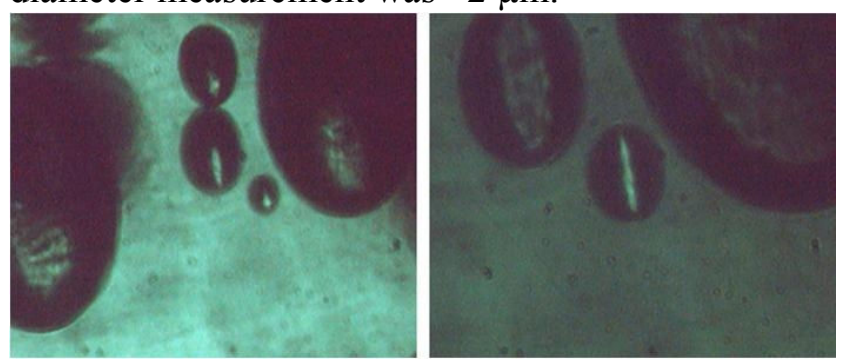

Fig. 4. Sample images of the bubbles.

\section{Results and Discussion}

\subsection{Results with Chalk specimens for Kidney Stone Simulation}

During the experiments, in order to measure the penetration effect of cavitation, pieces of chalk were used as specimens. Chalk is a good test material as it is relatively easy to 
differentiate even the smallest penetration differences between consecutive runs. Moreover, since chalk is made of gypsum (calcium sulfate) its material is similar to the content of kidney stone. The depth of penetration was measured after placing the piece of chalk 1-2 $\mathrm{mm}$ downstream just in front of the outlet. All consecutive test runs were made under the same working conditions. The depths were measured with a microscope after marking the deepest point of the chalk piece without further damaging. It was relatively easy to measure the depth since the system outlet was focused on the upper half of the chalk, making it possible to observe the deepest point under the microscope.

In Fig. 5a, the penetration depth is displayed as a function of time. As expected, the penetration of the chalk medium increases with time. It is also evident that the distance between the microprobe and the specimen is an important parameter. The penetration depth is larger for closer distances due to stronger bubble-specimen surface interactions. The data for penetration depth were converted to the mass removed by hydrodynamic cavitation assuming the erosion to have a hemispherical shape. This assumption was borne out by observation of the exposed surfaces. The same trends as in Fig. 5a are valid for the dependence of the removed mass on time (Fig. 5b). Removal rates of about 40 $\mathrm{mg} / \mathrm{min}$ and $35 \mathrm{mg} / \mathrm{min}$ are achieved for distances between the microprobe and specimen surfaces of $1 \mathrm{~mm}$ and $2 \mathrm{~mm}$, respectively. The rapid erosion in the specimen's surface proves the effectiveness of the proposed method in kidney stone treatments. A typical sample of surface erosion caused by bubbly cavitation is shown in Fig. 5c.

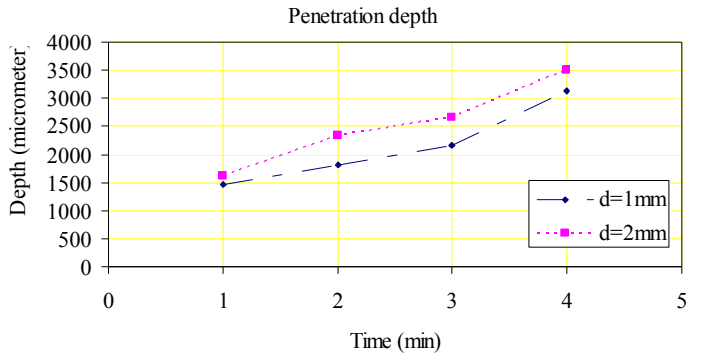

Fig. 5a. Penetration depth data [d in $\mu \mathrm{m}]$

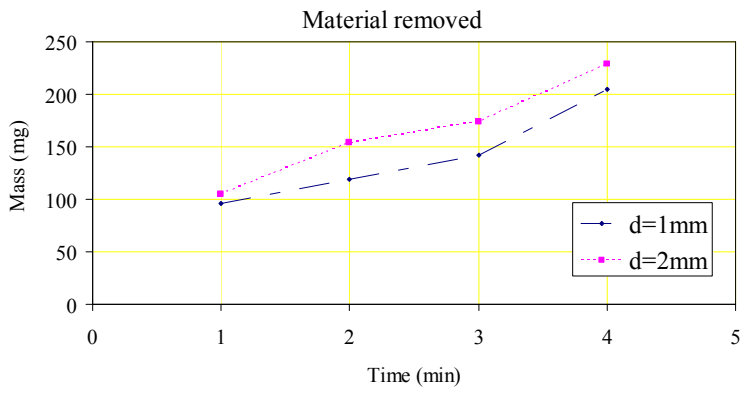

Fig. 5b. Dependence of Material Removed from Chalk on microprobe/specimen distance and time of exposure [mass in $\mathrm{mg}$ ]

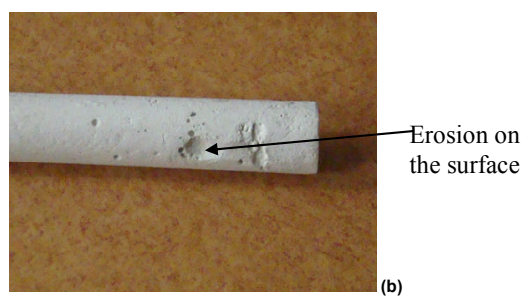

Fig. 5c. Erosion on chalk surface after exposure to bubbly cavitation

\subsection{Cavitation Experiments with Cancerous Cells}

Material and Methods:

The same experimental method and apparatus were used to initiate bubbly cavitation for these experiments. The details about cavitation induced cell-deaths are shown below to reveal the potential of hydrodynamic cavitation in biomedical treatment.

\section{Cell Culture for cavitation experiments:}

Jurkat, acute leukemia T cells, and U937, human myelomonocytic cell lines, were obtained from the American Type Culture Collection (ATCC). These cells were cultured in RPMI-1640 medium containing 10\% Fetal Bovine Serum, $2 \mathrm{mM}$ L-glutamine, 100ug penisilin/ 100U streptomycin, 55uM $\beta$ mercaptoethanol at $37^{\circ} \mathrm{C}$ in a humid $5 \% \mathrm{CO} 2$ $95 \%$ air environment. Medium was replaced every 2 to 3 days.

Experimental set-up to create singlecavitation bubbles in a physiological solution:

For the time-dependent treatments, the 
cells were cultured in $75 \mathrm{~cm}^{2}$ flasks and maintained at a cell concentration $3 \times 106$ cells $/ \mathrm{ml}$. All equipment were sterilized with $70 \%$ ethanol and a physiological solution, phosphate buffered saline (PBS), was used as a liquid environment. Cells were treated with bubbly cavitation under an inlet pressure of about $950 \mathrm{kPa}$ (inlet pressure was increased until a cavitating flow pattern was obtained) for $30 \mathrm{sec} ., 1,2,3,5$ and $10 \mathrm{~min}$, while the outlet pressure (pressure in the solution) was kept as atmospheric pressure.

\section{Cell Death Analysis:}

After the cavitation experiment, the cells were centrifuged at $300 \mathrm{~g}$ for $5 \mathrm{~min}$., carefully washed and resuspended with a fresh medium. Cell viability was determined by trypan blue exclusion and counted under light microscopy at $0,16,24$ and 48 hours.

Cavitation-induced cell death:

Effects on cell viability and especially the induction of apoptosis have been reported for cancerous human lymphocytes exposed to cavitation bubbles driven with a continuous ultrasound wave generator. Here, for the study of cell death, viability of Jurkat cells after their exposure to hydrodynamically produced bubbly cavitation is concomitantly assessed using trypan blue exclusion up to 48 $\mathrm{h}$. This is compared to control samples, cultured in same conditions but not submitted to a cavitation event. Figures 6 and 7 present the results as a percentage of total cell number for both series of samples (live and death) at 30 sec. 1, 2, 3, 5 and $10 \mathrm{~min}$. From the data presented in Figs. 6 and 7, it can be concluded that overall viability of Jurkat cells exists up to $10 \mathrm{~min}$. The results just after the exposure to bubbly cavitation are comparable to that observed in control for short time periods. Interestingly, a dramatic decrease in cell viability is subsequently observed for the treated samples at $24 \mathrm{~h}$, which can be explained with the programmed cell death, apoptosis, or necrosis. Indeed, attempts to carry out a cavitation-induced cell death have been successful.
Cavitation-induced cell death: Cell viability

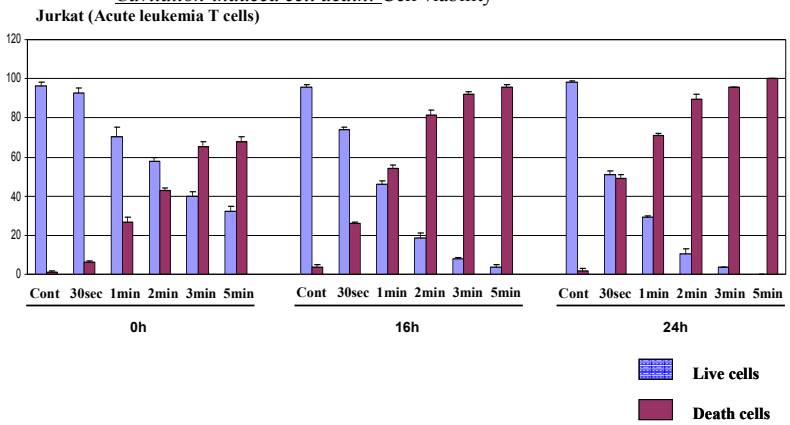

Fig. 6: Cell viability of Jurkat cells.

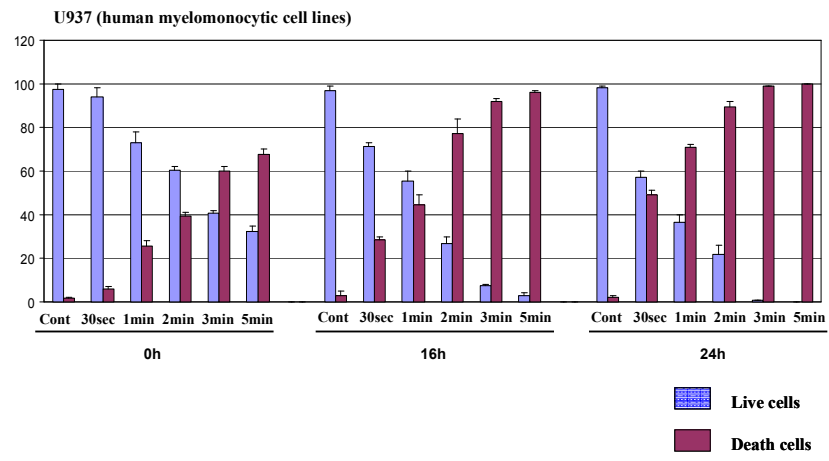

Fig. 7: Cell viability of U-937 cells.

\section{Conclusions}

The potential of hydrodynamic cavitation for use in medical treatment was explored. Two sets of experiments were undertaken, using firstly chalk specimens, similar in properties to kidney stones, and secondly cell cultures containing cancerous cells. Both target materials were exposed to bubbly cavitation hydrodynamically generated by a micro orifice, and the changes in the targets were carefully examined. The following conclusions were drawn from the results of the respective experiments:

- Bubbly cavitation could successfully cause significant and rapid erosion to exposed chalk surface areas. The combination of successful erosion and the ability finely to control the process demonstrates that microscale hydrodynamic cavitation is an alternative treatment method for removal of kidney stones

- Micro scale bubbly cavitation caused 
cell deaths in two different cell cultures containing cancerous cells. With increasing exposure time more cell deaths took place. In addition, dramatic decrease in cell viability was observed with time after such exposure. These findings clearly show the capability of hydrodynamic bubbly cavitation to cancer treatment provided fine targeting can be achieved.

\section{Acknowledgements}

This work was supported by Sabanc1 University Internal Grant for Research Program under Grant FRG-C47004. Undergraduate student support from Faculty of Natural Sciences and Engineering of Sabanc1 University is also gratefully appreciated. Finally the clinically-defined cultures were obtained from the American Type Culture Collection (ATCC).

\section{Nomenclature}

$\begin{array}{ll}\mathrm{A} & \text { Area } \\ \mathrm{D} & \text { Distance } \\ \mathrm{m} & \text { Mass } \\ \mathrm{P} & \text { Static pressure } \\ \mathrm{R} & \text { Bubble radius } \\ \mathrm{T} & \text { Temperature } \\ \mathrm{t} & \text { Time } \\ V & \text { Velocity } \\ \text { Greek } & \text { Letters } \\ \mu & \text { Viscosity } \\ \rho & \text { Density } \\ \sigma & \text { Cavitation number }\end{array}$

\section{Subscripts}

$\begin{array}{ll}\text { o } & \text { Initial value } \\ \infty & \text { Value far from the bubble } \\ 1 & \text { Pertaining to the liquid phase } \\ \text { v } & \text { Pertaining to the vapor phase } \\ \text { th } & \text { Pertaining to the orifice throat }\end{array}$

\section{References}

[1] Brennen, C. E., 2005. Fundamentals of multiphase flow., Cambridge University Press, New York, USA.

[2] Crowe, C. T., 2006. Multiphase flow handbook., CRC Taylor and Francis.

[3] Plesset, M. S., 1949. The dynamics of cavitation bubbles. J. Appl. Mech 16:277

[4] Brennen, C. E., 1995. Cavitation and bubble dynamics., Oxford University Press

[5] Goldenstedt, C., Melodelima, D., Mithieux, F., Chesnais, S., Theillère Y and Cathignol, D., 2004. Cavitation enhances treatment depth when combined with thermal effect using a place ultrasound transducer: an in vivo study., 2004 IEEE Ultrasonics Symposium 709-712

[6] Melodelima, D. and Cathignol, D., 2004. Cancer treatment by ultrasound: increasing the depth of necrosis. Applied Physics Letters 84:5365-5367

[7] Moholkar, V. S. and Pandit, A. B., 2001. Numerical investigations in the behavior of one-dimensional bubbly flow in hydrodynamic cavitation. Chemical Engineering Science 56:1411-1418

[8] Moholkar, V. S. and Pandit A. B., 2001. Modeling of hydrodynamic cavitation reactors: a unified approach. Chemical Engineering Science. 56:6295-6302

[9] Kumar, K. S. and Moholkar, V. S., 2007. Conceptual design of a novel hydrodynamic cavitation reactor. Chemical Engineering Science. 62:2698-2711

[10] Loraine, G., 2007. Liquid oxidation: Hydrodynamic cavitation for cost effective oxidation. Filtration \& Separation 44:16-18

[11] Arrojo, S. and Nenito, Y. 2008. A theoretical study of hydrodynamic cavitation. Ultrasonics Sonochemistry. 15:203-211

[12] Mishra, C. and Peles, Y. 2005. Cavitation in flow through a micro-orifice inside a silicon microchannel. Phys Fluids 17:013601

[13] Schneider, B., Koşar, A., Kuo, C. J., Mishra, C., Cole, G. S., Scaringe, R. P., and Peles, Y. 2006. Cavitation enhanced heat transfer in microchannels. Journal of Heat Transfer 128:1293-1301

[14] Mishra, C. and Peles, Y. 2005. Size scale effects on cavitating flows through 
microorifices entrenched in rectangular microchannels. Journal of Microelectromechanical Systems 14:987-999 [15] Mishra, C. and Peles, Y. 2006. An experimental investigation of hydrodynamic cavitation in micro-venturis. Phys Fluids 18:103603

[16] Mishra, C. and Peles, Y. 2005. Flow visualization of cavitating flows through a rectangular slot micro-orifice ingrained in a microchannel. Phys Fluids 17:113602

[17] Thüroff, S., Chaussy, C., Vallancien, G., Wieland, W., Kiel, H. J., Le Duc, A., Desgrandchamps, F., De La Rosette, J. J., and Gelet, A., 2003. High intensity focused ultrasound and localized prostate cancer: efficacy results from the European multicentric study. J Endourol 17:673-677

[18] Oosterhof, G. O. N., Comel, E. B., and Smits, H. J. 1996. The influence of high energy shock waves on the development of metastasis. Ultrasound in Medicine and Biology. 22:339-344 